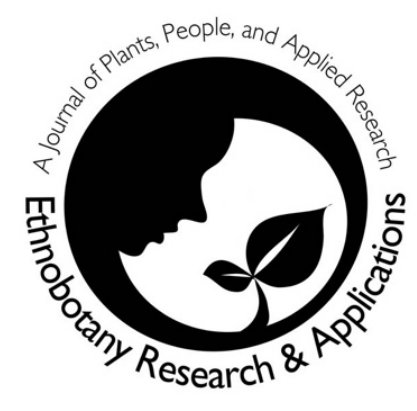

\title{
Medicinal Plants Used in COVID-19 Pandemic in Buddhabhumi Municipality, Kapilvastu, Nepal
}

\author{
Vivek Thapa Chhetri, Pabitra Jha and Surya Kumar \\ Maharjan
}

\author{
Correspondence \\ Vivek Thapa Chhetri ${ }^{{ }^{*}}$, Pabitra Jha1, Surya Kumar Maharjan ${ }^{2}$ \\ ${ }^{1}$ Tribhuvan University, Institute of Forestry, Pokhara Campus, Pokhara 33700, Nepal \\ ${ }^{2}$ Tribhuvan University, Institute of Forestry, Hetauda Campus, Hetauda 44107, Nepal \\ *Corresponding Author: thapavivek777@gmail.com
}

Ethnobotany Research \& Applications 22:26 (2021)

\section{Research}

\begin{abstract}
Background. Currently, the Coronavirus disease (COVID-19) pandemic is posing a serious threat to global health. Although approved COVID-19 vaccines have been claimed to provide a high degree of protection against getting seriously ill and dying from the disease, they are not in the approach of all people especially those from the least developed countries like Nepal. Desperation within people has led to increased use of medicinal plants to cure the miscellaneous symptoms of COVID-19 and to prevent infection without proper knowledge of their phytochemical and pharmacological properties.
\end{abstract}

Methods. Based on the online and interpersonal inquiries with 59 COVID-19 survivors and 111 inhabitants of Buddhabhumi Municipality, Kapilvastu, Nepal, the ethnomedicinal data were collected and summarized using descriptive statistics like frequency, percentage tables, and graphs. For each medicinal plant species, the frequency of citation (FC) and relative frequency of citation (RFC) were calculated along with a radar chart for the top 15 cited species with their phytochemical and pharmacological properties.

Results. This study documented 41 species of medicinal plants belonging to 24 different families and 37 genera were used. Herbs were predominant among other life-forms and leaves were highly used among other plant parts. Turmeric (Curcuma longa, with the highest frequency of citation of 0.80 ) was the most frequently used species. Most of the respondents (44\%) obtained medicinal plants from their home gardens. Family, friends, and neighbors were the primary sources of information the respondents followed. Interestingly, all of the 15 most frequently cited medicinal plants were reported to have both antiviral and anti-inflammatory properties and 14 of them were proclaimed to be immunomodulatory.

Conclusion: Therefore, we conclude from the survey and available literatures that medicinal plants play a significant role against viral infections.

Keywords. Anti-inflammatory, Antiviral, Immunomodulatory, Pharmacology, Phytochemical

\section{Background}

The worldwide health crisis caused by SARS-CoV-2, the origin of the Coronavirus disease (COVID-19) outbreak, has reminded us that the globe is no longer functioning, presumably business as usual. After the Severe Acute 
Respiratory Syndrome Coronavirus (SARS-CoV) in 2003 (De et al. 2016, Lee et al. 2003, Pieris et al. 2003) and the Middle East Respiratory Syndrome Coronavirus (MERS-CoV) in 2012 (Alagaili et al. 2014, Memish et al. 2013, Zaki et al. 2012), COVID-19 is the third novel coronavirus to cause a large-scale epidemic in the twenty-first century. The COVID-19 outbreak started in December 2019 (Sheeran et al. 2020) and proceeded differently in numerous regions. As of $20^{\text {th }}$ August 2021, 4,400,284 individuals worldwide have lost their lives due to COVID infection (WHO 2021). COVID-19 has triggered several endeavors to prevent disease transmission in addition to preventive measures (Lake 2020, Salathe et al. 2020). Few vaccines have been permitted for emergency use and to provide a high degree of protection against getting seriously ill and dying from the disease (Li et al. 2021). However, not everyone has access to those vaccines (Saad-Roy et al. 2020). Particularly, people from the least developed countries like Nepal have limited access to vaccines. Besides, as the vaccines were developed over a very short period, their long-term efficacy and side effects are yet to be confirmed (Forni \& Mantovani 2021). In this context, provided herbal medicines have been used for the treatment of viral infections since time immemorial (Wang et al. 2020), medicinal plants are useful as an alternative for symptomatic treatment and boosting the immune system (Sharma et al. 2017).

Lifestyle, nutrition, age, gender, health conditions, and environmental variables all have a pivotal role in susceptibility to COVID-19 severity (Gasmi et al. 2020). There is a lot of testimony to support the efficacy of medicinal plants in treating viral infections (Zhang et al. 2020). Still, it is challenging to state how well medicinal plants work since they're usually combined with allopathy (Vandebroek et al. 2020). Traditional Chinese herbal medicine was revealed to be an efficient therapy to prevent and cure COVID-19, which proudly endorses herbal medication as an alternative remedy (Benarba \& Pandiella 2020, Hossain et al. 2020, Li et al. 2020). Screening analysis of hundreds of Chinese medicinal herbs revealed the presence of anti-SARS-CoV property in the plant extracts (Vandebroek et al. 2020). Many bioactive compounds have been tested for their antiviral activity against human viruses (Adhikari et al. 2021, Bosquet et al. 2021). Despite having little knowledge about the chemical properties of medicinal plants, they are prescribed because of their claimed efficacy and availability (Mohammad et al. 2018). Consequently, the demand for medicinal plants is increasing and has become an appropriate alternative treatment option (Pandey et al. 2020, Vandebroek et al. 2020).

Currently, Nepal is experiencing the second wave of COVID-19 and the cases are skyrocketing. As of $22^{\text {nd }}$ August 2021, 10,509 individuals have lost their lives due to the COVID-19 infection in Nepal, and the death number is still soaring (MOHP 2021). Given the limited access to modern medicine, i.e., only $17 \%$ of city-dwellers have access to modern medicine (Adhikari et al. 2019) and about 5.1\% of the country's population have been vaccinated till $13^{\text {th }}$ June (Reuters 2021), an increasing number of people are exploring alternatives of medicinal plant-based therapeutics. With the rise in unsubstantiated efficacy claims circulating on social media, many misinterpretations of the use of medicinal plants to treat or prevent COVID-19 are spreading throughout Nepal. Nepal is considered the native land of medicinal plants (Pandit \& Singh 2020) and has a tradition of using them (Kunwar et al. 2010), but the irony is that the study and documentation of medicinal plants used during the COVID-19 period in Nepal are limited. In this context, this study aims to document (1) the medicinal plants being used for COVID-19 treatment and prevention (2) the parts of the plants used; (3) their source; (4) the source of information people follows to use them; (5) people's perception about the efficacy of medicinal plants in curing COVID-19 symptoms or preventing infections; and (6) the phytochemical and pharmacological properties of the medicinal plants being used. By doing so, this study will not only explore information that can be used by the general public, and prospective COVID-19 patients but also contribute to the knowledge base that can be used by the global scientific community to formulate novel drugs and select clinical trials for COVID-19 control and prevention.

\section{Materials and Methods}

\section{Study area}

This study focused on Buddhabhumi Municipality which is one of the most severely affected municipality by COVID19 in the Kapilvastu district (Health Office Kapilvastu 2021) of Nepal. In terms of area, it is the largest (366.67 sq km) of the ten municipalities in the Kapilvastu District. It is inhabited by 64,949 individuals (11,509 households) (Central Bureau of Statistics 2018). As of $16^{\text {th }}$ Apr, 2021, out of 250 infected individuals, 240 have recovered from COVID-19 infection in the municipality (Health Office Kapilvastu 2021). The map of the study area is presented here (Figure 1) 


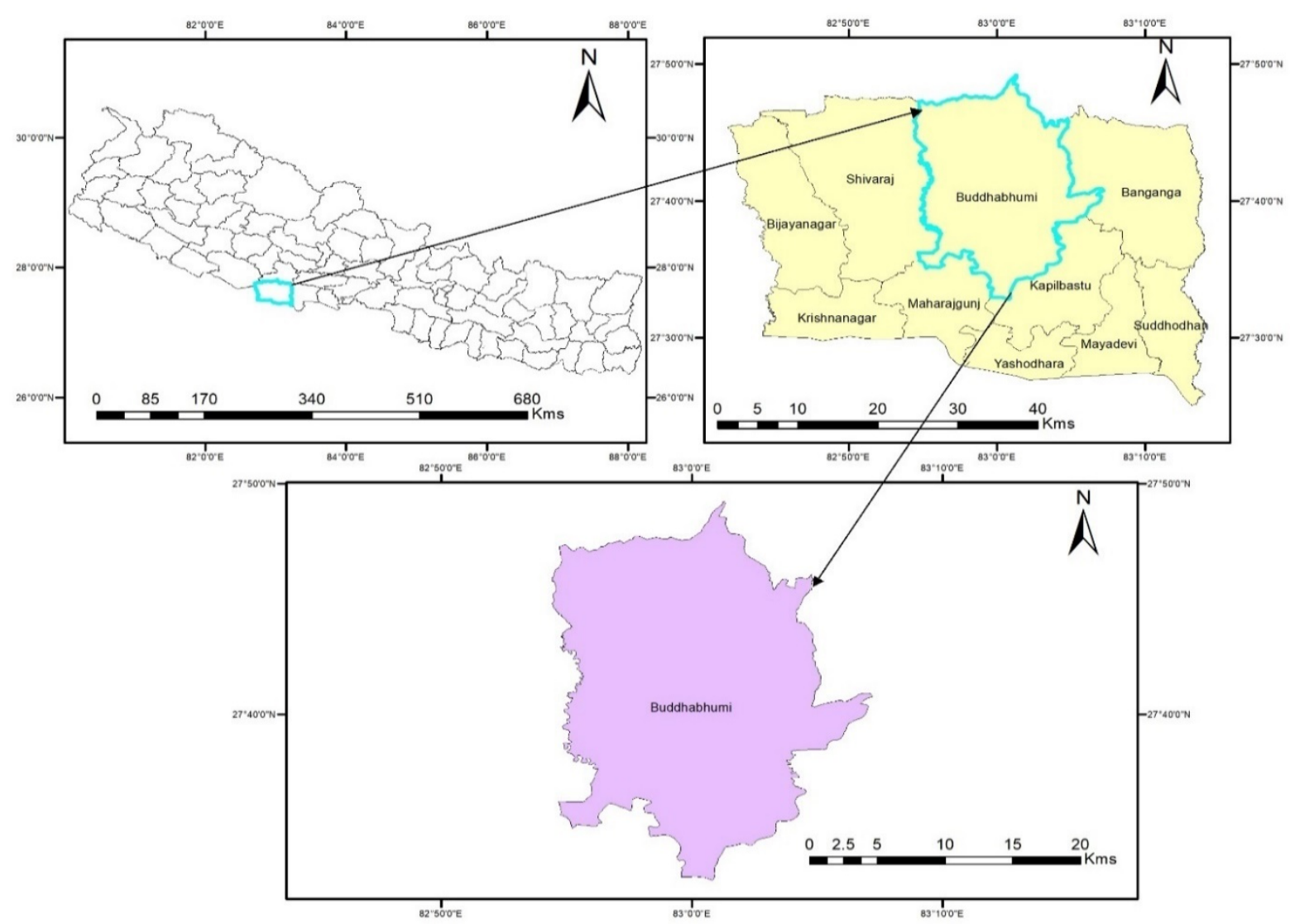

Figure 1. Study area map of Buddhabhumi Municipality. The top left panel shows the location of the Kapilvastu district - the study district and the top right panel shows that of Budhhabhumi Municipality - the studied municipality.

\section{Methods of data collection}

Given the current mobility restrictions, we used online inquiries to compile the necessary data. A set of questionnaire forms including close- and open-ended questions was designed in Google Forms Developer. Initially, the Google Form was tested among 25 individuals to validate and comprehend response rates. Throughout our research, we adhered to the norms of the International Society of Ethnobiology and included a written consent message for everyone we reached out with (International Society of Ethnobiology 2006). The Google Form was circulated to 150 individuals of Buddhabhumi Municipality through social media between $5-19^{\text {th }}$ May 2021 . Out of which, 90 individuals (60\%) from 10 wards of the municipality filled out the form and provided information as per the questions mentioned there. In addition to the Google survey, another 60 individuals whose contact details were obtained from the Municipality office were telephoned to collect the data. Furthermore, 20 individuals were interviewed in person, of course following the COVID-19 health standards, using a printed questionnaire. Altogether, 170 individuals were surveyed which covers both COVID-19 survivors (59) and not infected individuals (111). The sample COVID-19 survivors represent $24.58 \%$ of the total COVID-19 survivors in the Buddhabhumi Municipality. Of the total 170 respondents, $63.52 \%$ were males and $36.48 \%$ were females. Most of them were from age class $18-30$ years (35.29\%) and had completed their higher secondary level education (27.64\%).

\section{Identification of Species}

The documented plant information was collected from the informants and validated their presence and use in COVID-19 treatment with the local healers. We followed TROPICOS (https://www.tropicos.org/) for the identification of scientific names, the authority of species, and their family of local species used by the respondents.

\section{Statistical Analysis}

The collected data were entered into MS Excel 2007 and summarized using descriptive statistics like frequency, percentage tables, and graphs. For each medicinal plant species, the frequency of citation (FC) and relative frequency of citation (RFC) was calculated using the following formula developed by Tardio \& Pardo-de-Santayana 2008: 


$$
R F C=\frac{F C}{N}
$$

Wwhere $R F C=$ relative frequency of citation; $F C=$ number of respondents who reported the use of medicinal plant species; and $N=$ total number of respondents took part in the survey

Based on the RFC, the top 15 medicinal plant species were identified. For the top 15 medicinal plant species, their families, phytochemical, and pharmacological details are listed based on the published literature to verify their use against viral infection.

\section{Methodological limitation}

Considering the current situation with limited mobility, we combined an online survey with some interpersonal interviews in this study. Since only the middle-aged educated respondents could access and respond to the online Google Form, it might have introduced some bias.

\section{Results}

\section{Medicinal plants used}

Of the 170 respondents, 150 (88\%) respondents reported that they used medicinal plants during the COVID-19 pandemic (Figure 2). Of the 150 respondents that used medicinal plants, 87 (51\%) used only medicinal plants, 63 (37\%) others used them along with allopathic medicine while 6 (4\%) used allopathic medicine alone and 14 (8\%) used none of them. The majority 49 (29\%) of the respondents used 8 to 12 species of medicinal plants (Figure 3 ). In total, 41 species of medicinal plants belonging to 24 families and 37 genera were used (Table 1). Among them, the most common families were Zingiberaceae (4 species), Apiaceae (4 species), Rutaceae (3 species), Lamiaceae (3 species), and Piperaceae (3 species). Similarly, the most dominant genera were Piper (3 species), Cinnamomum (2 species), Allium (2 species), and Curcuma (2 species). The majority of the medicinal plant species were herbs 19 (46\%), followed by trees $14(34 \%)$, climbers $4(10 \%)$, and shrubs 4 (10\%). For species, Curcuma longa $(0.80)$ was the most cited species and Camellia sinensis (0.01) was the least cited species (Table 1).

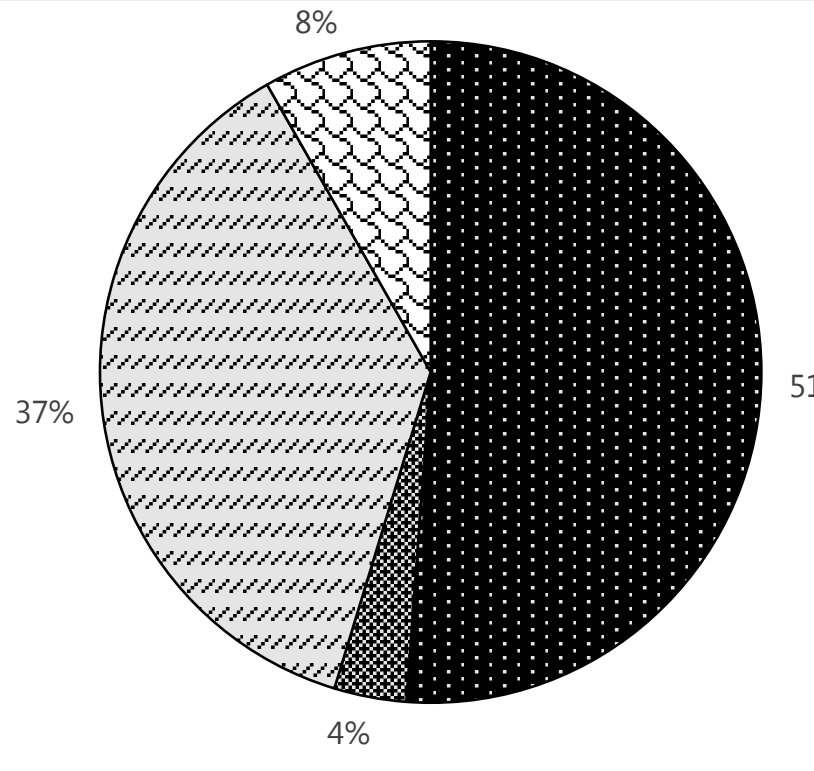

- Herbal treatment (Use of herbs and medicinal plants)

Allopathic treatment (Symptomatic medicine)

๑ Both

巴None

Figure 2. Curative and preventive treatment options followed by the respondents during the COVID-19 period. 


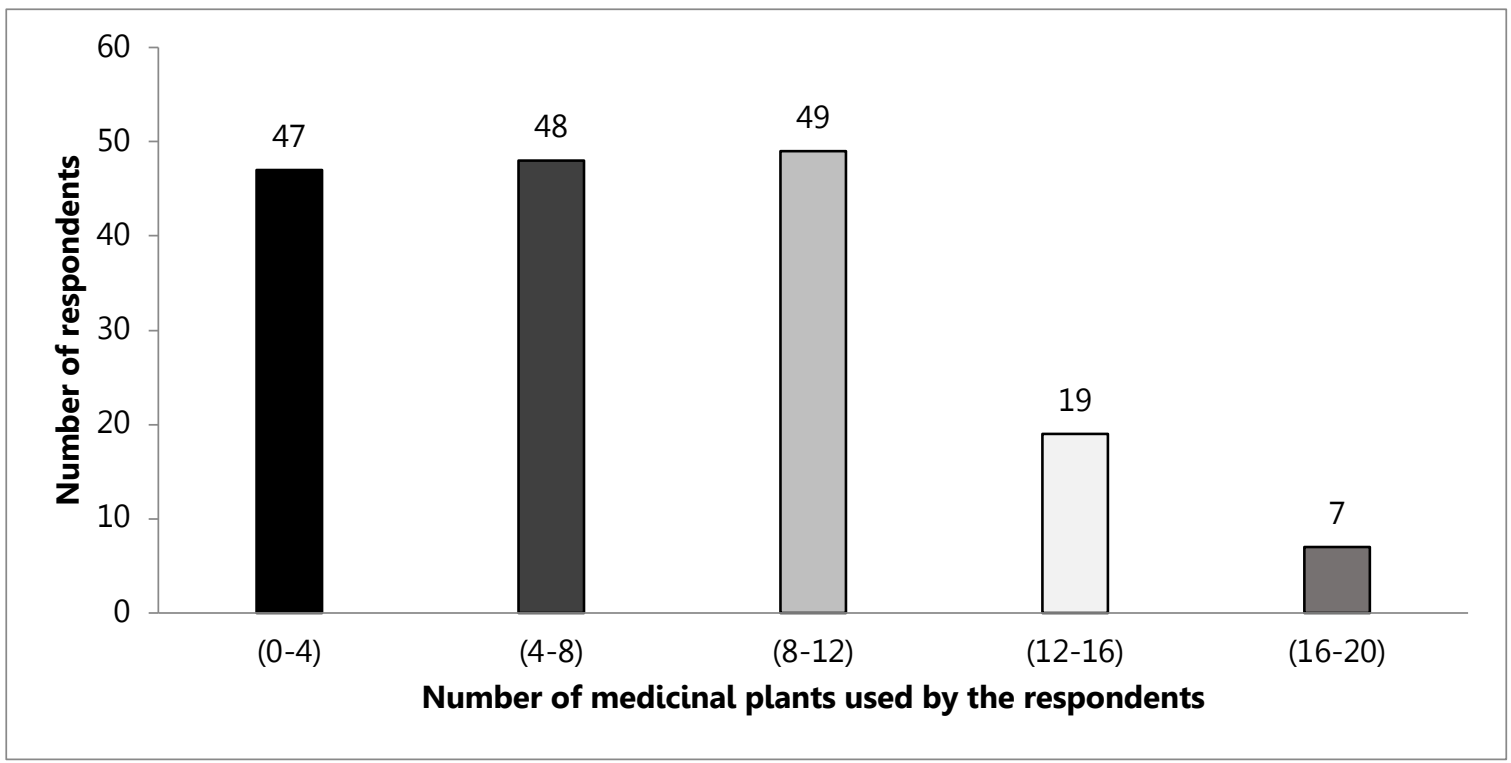

Figure 3. Number of medicinal plants used by the respondents during the COVID-19 period.

Parts of the medicinal plants used, their modes and frequency of use

For some medicinal plant species, entire plants were used, while for some others, specific parts were used. Leaves (37\%) were among the most predominantly used parts, followed by rhizome (22\%), seed (20\%), fruit (17\%), Bark (10\%), Stem and whole plant (5\%), Root and Flower (2\%) (Table 1). The most frequently applied process was decoction (to boil plant parts i.e., leaves, rhizome with water) and drink (Table 1). The frequency of medicinal plant use during the period of COVID-19 varied from often 42 (25\%) to rarely 20 (12\%) (Figure 4).

Table 1. List of medicinal plants used during the COVID-19 pandemic. Scientific names, their respective English names, local names, parts used, mode of use, frequency of citation (FC), and relative frequency of citation (RFC) are shown. Species are grouped by their families.

\begin{tabular}{|c|c|c|c|c|c|c|c|}
\hline Scientific name & English name & Local name & Habit & $\begin{array}{l}\text { Parts } \\
\text { used }\end{array}$ & Mode of use & FC & RFC \\
\hline \multicolumn{8}{|l|}{ Acanthaceae } \\
\hline Justicia adhatoda L. & Malabur nut & Asuro & $S$ & L & Decoction & 29 & 0.17 \\
\hline \multicolumn{8}{|l|}{ Acoraceae } \\
\hline Acorus calamus L. & Sweet flag & Bojho & $\mathrm{H}$ & $\mathrm{R}$ & Steam & 9 & 0.05 \\
\hline \multicolumn{8}{|l|}{ Amaryllidaceae } \\
\hline Allium cepa L. & Onion & Pyaaj & $\mathrm{H}$ & $R$ & Cook, Soup & 13 & 0.08 \\
\hline Allium sativum $\mathrm{L}$. & Garlic & Lasun & $\mathrm{H}$ & $\mathrm{Bu}$ & Soup, Paste & 20 & 0.12 \\
\hline \multicolumn{8}{|l|}{ Anacardiaceae } \\
\hline Mangifera indica $\mathrm{L}$. & Mango & Amp & $\mathrm{T}$ & L, B & Decoction & 13 & 0.08 \\
\hline \multicolumn{8}{|l|}{ Apiaceae } \\
\hline Centella asiatica (L.) Urb. & Water pennywort & $\begin{array}{l}\text { Ghodtapre / } \\
\text { Toprejhar }\end{array}$ & $\mathrm{H}$ & $R$ & Decoction & 18 & 0.11 \\
\hline Coriandrum sativum $\mathrm{L}$. & Coriander & Dhaniya & $\mathrm{H}$ & Se, L & $\begin{array}{l}\text { Decoction, } \\
\text { Paste } \\
\end{array}$ & 8 & 0.05 \\
\hline Cuminum cyminum L. & Cumin & Jeera & $\mathrm{H}$ & $\mathrm{Se}$ & Decoction & 8 & 0.05 \\
\hline $\begin{array}{l}\text { Trachyspermum ammi(L.) } \\
\text { Sprague }\end{array}$ & Thyme seed & Jwano & $\mathrm{H}$ & $\mathrm{Se}$ & Decoction & 111 & 0.65 \\
\hline \multicolumn{8}{|l|}{ Apocynaceae } \\
\hline Ceropegia pubescens Wall. & $\begin{array}{l}\text { Yellow Himalayan } \\
\text { ceropegia }\end{array}$ & $\begin{array}{l}\text { Mirke laharo / } \\
\text { simi laharo }\end{array}$ & $\mathrm{H}$ & St & Decoction & 3 & 0.02 \\
\hline \multicolumn{8}{|l|}{ Asteraceae } \\
\hline Artemesia indica Willd. & $\begin{array}{l}\text { Indianworm / } \\
\text { mugwort }\end{array}$ & Titepati & $\mathrm{H}$ & L & Decoction & 31 & 0.18 \\
\hline
\end{tabular}




\begin{tabular}{|c|c|c|c|c|c|c|c|}
\hline Scientific name & English name & Local name & Habit & \begin{tabular}{|l|} 
Parts \\
used
\end{tabular} & Mode of use & FC & RFC \\
\hline Spilanthes acmella (L.) L. & Toothache plant & Ban maratthi & $\mathrm{H}$ & $\mathrm{Fl}$ & Decoction & 18 & 0.11 \\
\hline \multicolumn{8}{|l|}{ Combretaceae } \\
\hline Terminalia chebula Retz. & $\begin{array}{l}\text { Chebulie } \\
\text { myrobalan/yellow } \\
\text { myrobalan }\end{array}$ & Harro & $\mathrm{T}$ & F, B & Decoction & 19 & 0.11 \\
\hline \multicolumn{8}{|l|}{ Equisetaceae } \\
\hline $\begin{array}{l}\text { Equisetum debile Roxb. ex } \\
\text { Vaucher }\end{array}$ & $\begin{array}{l}\text { Branched } \\
\text { horsetail }\end{array}$ & Kurkure & $\mathrm{H}$ & $R$ & Decoction & 2 & 0.01 \\
\hline \multicolumn{8}{|l|}{ Fabaceae } \\
\hline Trigonella foenum-graecum $\mathrm{L}$. & Fenugreek & Meethi & $\mathrm{H}$ & $\mathrm{Se}, \mathrm{L}$ & Paste & 9 & 0.05 \\
\hline \multicolumn{8}{|c|}{ Lamiaceae } \\
\hline Ocimum tenuiflorum $\mathrm{L}$. & Holy basil & Tulsi & $\mathrm{H}$ & L, Se & Decoction & 131 & 0.77 \\
\hline Mentha arvensis $\mathrm{L}$. & Pepper mint & Pudina & $\mathrm{H}$ & W & $\begin{array}{l}\text { Decoction, } \\
\text { Paste }\end{array}$ & 7 & 0.04 \\
\hline $\begin{array}{l}\text { Pogostemon benghalensis } \\
\text { (Burm. f.) Kuntze }\end{array}$ & $\begin{array}{l}\text { Bengal Shrub- } \\
\text { Mint }\end{array}$ & Rudilo & $\mathrm{T}$ & L & Decoction & 55 & 0.32 \\
\hline \multicolumn{8}{|l|}{ Lauraceae } \\
\hline $\begin{array}{l}\text { Cinnamomum tamala (Buch. - } \\
\text { Ham.) T. Nees \& Nee }\end{array}$ & Indian bay leaf & Tejpat & $\mathrm{T}$ & $L$ & Decoction & 25 & 0.15 \\
\hline $\begin{array}{l}\text { Cinnamomum zelyanicum } \\
\text { Blume }\end{array}$ & Cinnamon bar & Dalchini & $\mathrm{T}$ & B & Decoction & 19 & 0.11 \\
\hline \multicolumn{8}{|l|}{ Meliaceae } \\
\hline Azadirachta indica A. Juss. & Neem tree & Neem & $T$ & $L, B$ & Decoction & 38 & 0.22 \\
\hline \multicolumn{8}{|l|}{ Menispermaceae } \\
\hline Cissampelos pareira $\mathrm{L}$. & Velvet leaf & Gujargano & $\mathrm{C}$ & $R$ & Decoction & 4 & 0.02 \\
\hline $\begin{array}{l}\text { Tinospora cordifolia (Willd.) } \\
\text { Miers }\end{array}$ & $\begin{array}{l}\text { Heart-leaved } \\
\text { moonseed }\end{array}$ & $\begin{array}{l}\text { Gurjo / Giloy / } \\
\text { Guduchi }\end{array}$ & C & St & Decoction & 135 & 0.79 \\
\hline \multicolumn{8}{|l|}{ Myristicaceae } \\
\hline Myristica fragrans Houtt. & Nutmeg & Jaiphal & $T$ & $\mathrm{Se}$ & Decoction & 3 & 0.02 \\
\hline \multicolumn{8}{|l|}{ Myrtaceae } \\
\hline Psidium guajava $\mathrm{L}$. & Guava & Belauti / Amba & $T$ & $\mathrm{~L}$ & Decoction & 56 & 0.33 \\
\hline $\begin{array}{l}\text { Syzygium aromaticum (L.) } \\
\text { Merr. \& L.M. Perry }\end{array}$ & Clove & Lwang & $T$ & $F$ & Raw, Paste & 16 & 0.09 \\
\hline \multicolumn{8}{|l|}{ Phyllanthaceae } \\
\hline Phyllanthus emblica L. & \begin{tabular}{|l|} 
Indian gooseberry \\
\end{tabular} & Amala & $\mathrm{T}$ & $\mathrm{F}$ & $\begin{array}{l}\text { Chew, Powder } \\
\text { taken with } \\
\text { honey }\end{array}$ & 23 & 0.14 \\
\hline \multicolumn{8}{|l|}{ Piperaceae } \\
\hline Piper cubeba L. f. & Java pepper & Sheetel chini & $T$ & W & Decoction & 9 & 0.05 \\
\hline Piper longum $\mathrm{L}$. & Long pepper & Pipla & $\mathrm{C}$ & $\mathrm{F}$ & Decoction & 29 & 0.17 \\
\hline Piper nigrum L. & Black pepper & Marich & C & $\mathrm{F}$ & Decoction & 74 & 0.44 \\
\hline \multicolumn{8}{|l|}{ Poaceae } \\
\hline Cynodon dactylon (L.) Pers. & $\begin{array}{l}\text { Bermuda/Devile } \\
\text { grass }\end{array}$ & Dubo & $\mathrm{H}$ & $R$ & Decoction & 3 & 0.02 \\
\hline \multicolumn{8}{|l|}{ Rutaceae } \\
\hline Aegle marmelos (L.) Correa & Indian bael & Bel & $T$ & $\mathrm{~L}$ & Decoction & 29 & 0.17 \\
\hline Citrus limon (L.) Osbeck & Lemon & Kagati & $T$ & $F, L$ & Decoction & 89 & 0.52 \\
\hline Zanthoxylum armatum DC. & \begin{tabular}{|l|} 
Nepal \\
pepper/prickly \\
ash \\
\end{tabular} & Timur & $\mathrm{S}$ & $\mathrm{F}$ & Soup, Paste & 28 & 0.16 \\
\hline \multicolumn{8}{|l|}{ Sapindaceae } \\
\hline Litchi chinensis Sonn. & Litchi & Lychee & $T$ & $\mathrm{~L}$ & Decoction & 3 & 0.02 \\
\hline
\end{tabular}




\begin{tabular}{|c|c|c|c|c|c|c|c|}
\hline Scientific name & English name & Local name & Habit & $\begin{array}{l}\text { Parts } \\
\text { used }\end{array}$ & Mode of use & FC & RFC \\
\hline \multicolumn{8}{|l|}{ Solanaceae } \\
\hline Withania somnifera (L) Dunal & Winter cherry & Ashwagandha & $S$ & Rt, Se, L & $\begin{array}{l}\text { Decoction, } \\
\text { Powder taken } \\
\text { with milk }\end{array}$ & 25 & 0.15 \\
\hline \multicolumn{8}{|l|}{ Theaceae } \\
\hline Camellia sinensis $(\mathrm{L})$ Kuntze & Tea & Chiya & $S$ & $\mathrm{~L}$ & Raw & 2 & 0.01 \\
\hline \multicolumn{8}{|l|}{ Zingiberacea } \\
\hline Amomum cardamomum L. & $\begin{array}{l}\text { Green } \\
\text { cardamomum }\end{array}$ & Sukmel & $\mathrm{H}$ & $\mathrm{Se}$ & Decoction & 2 & 0.01 \\
\hline Curcuma caesia Roxb. & Black turmeric & Kalo haledo & $\mathrm{H}$ & $\mathrm{R}$ & Decoction & 2 & 0.01 \\
\hline Curcuma longa $\mathrm{L}$. & Turmeric & Haldi/Beshar & $\mathrm{H}$ & $\mathrm{R}$ & $\begin{array}{l}\text { Decoction, } \\
\text { Powder taken } \\
\text { with milk }\end{array}$ & 136 & 0.80 \\
\hline Zingiber officinale Roscoe & Ginger & Aduwa & $\mathrm{H}$ & $R$ & $\begin{array}{l}\text { Decoction, } \\
\text { Paste }\end{array}$ & 73 & 0.43 \\
\hline
\end{tabular}

Habit: $\mathrm{H}=$ Herb, $\mathrm{S}=$ Shrub, $\mathrm{C}=$ Climber, $\mathrm{T}=$ Tree; Parts used: $\mathrm{L}=$ Leaf, $\mathrm{R}=$ Rhizome, Se=Seed, $\mathrm{F}=\mathrm{Fruits}, \mathrm{St}=\mathrm{Stem}, \mathrm{Fl}=$ Flower, $\mathrm{W}=$ Whole plant, $\mathrm{B}=$ Bark, $\mathrm{Bu}=\mathrm{Bulb}, \mathrm{Rt}=\mathrm{Root}$

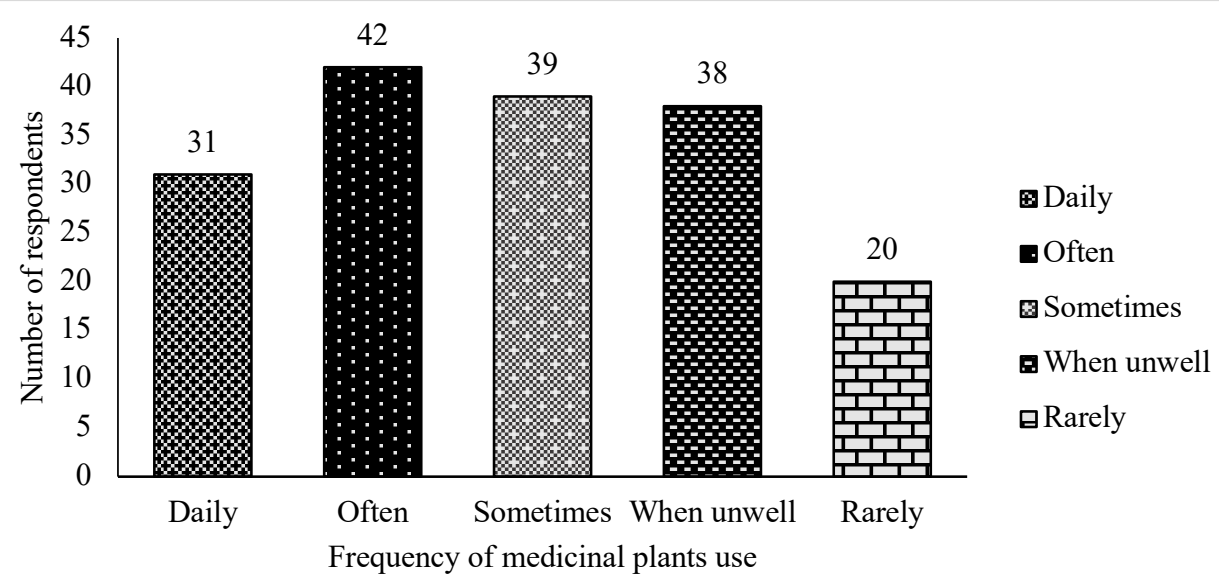

Figure 4. Frequency of use of medicinal plants.

\section{Sources of medicinal plants}

The majority of the respondents stated they used medicinal plants available in their home gardens 192 (44\%), others either purchased them from the nearest market 159 (37\%), herbal shop 17 (4\%) or collected them from the nearest forest areas 64 (15\%) (Figure 5).

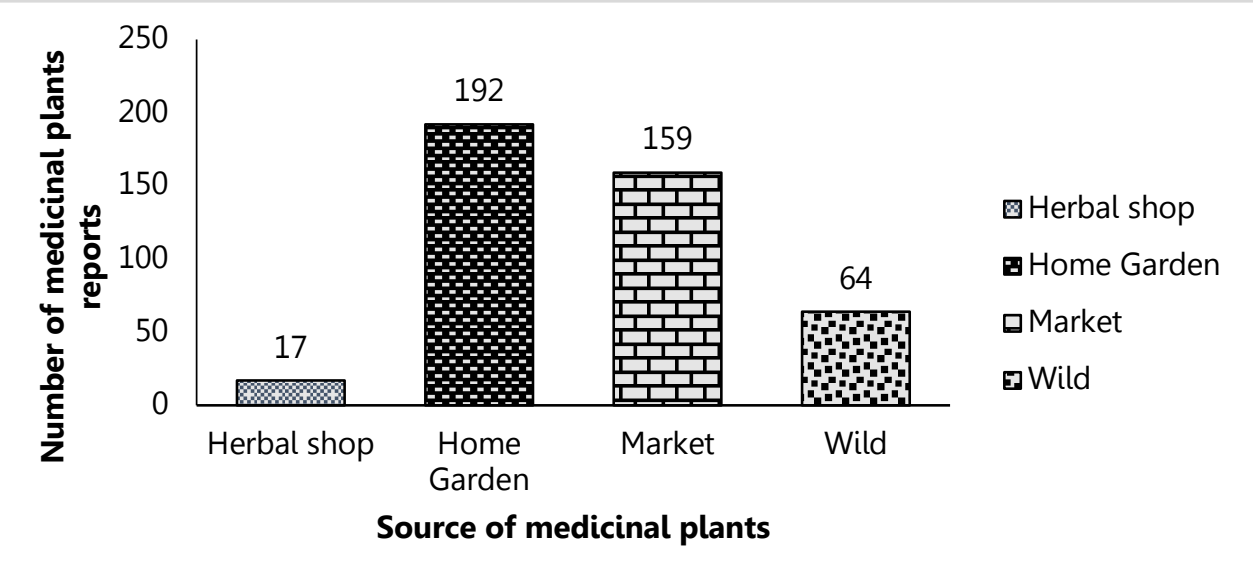

Figure 5. Source of medicinal plants. 
Information sources of people followed to use medicinal plants

The majority (162) of the respondents received information about the possibility of using medicinal plants to cure or prevent COVID-19 infection from their family/friends/neighbors followed by the internet (66) (Figure 6). Others relied on other different sources such as books, TV/radio, local health workers, or other sources for information.

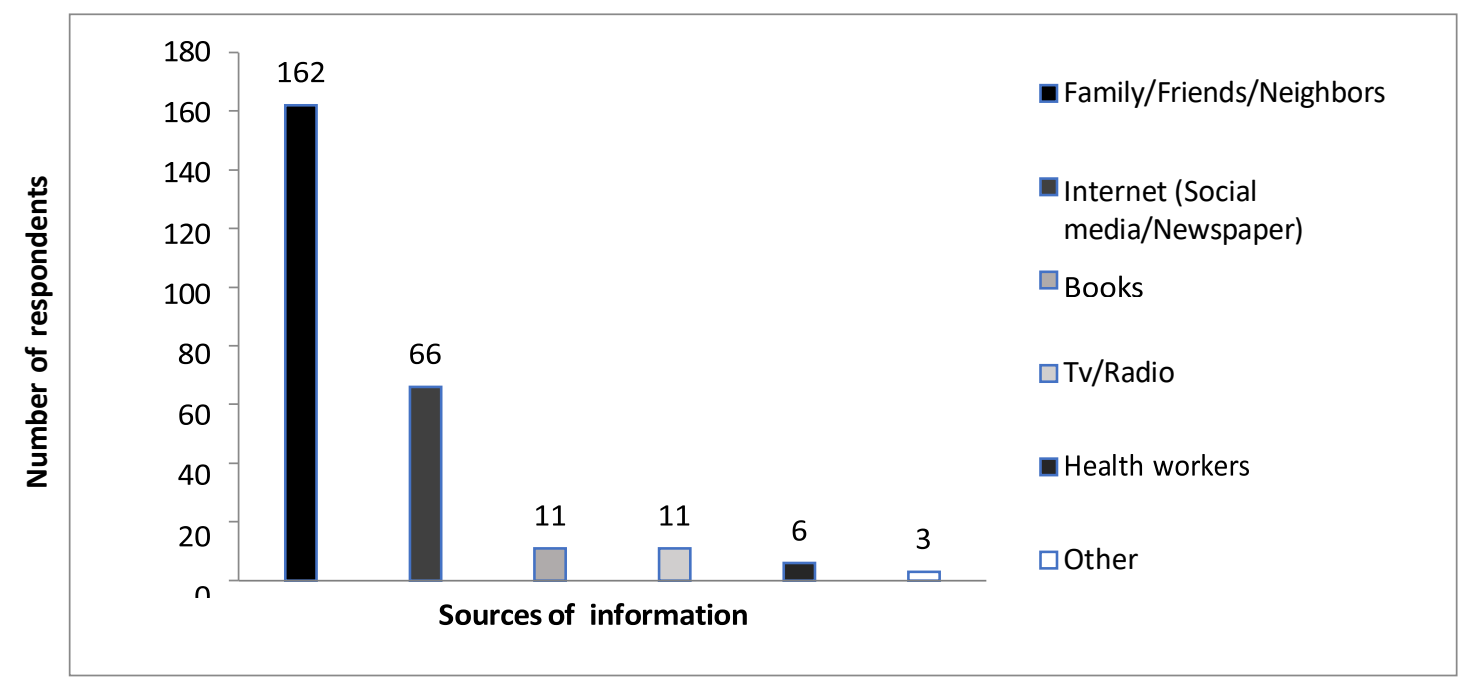

Figure 6. Sources of information that people followed to use medicinal plants.

People's perception about the efficacy of medicinal plants in preventing COVID-19 infection

Of the 59 COVID-19 survivors, $90 \%$ (53) of the survivors reported that they used medicinal plants for treating COVID19 symptoms and $81 \%$ (43) of those COVID-19 survivors reported that medicinal plants helped to cure the COVID19 related symptoms. The survivors consumed medicinal plants primarily for 11 to 15 days to recover from COVID19 (Figure 7). Paying it forward, each of the 22 (42\%) of the satisfied COVID-19 survivors had suggested more than ten individuals in their networks to use medicinal plants (Figure 8). Of the 170 respondents, the majority (66\%) of the respondents expressed their belief that medicinal plants can enhance their immunity against viruses, but they were doubtful about their efficacy in preventing COVID-19 infection. In contrast, $18 \%$ of the respondents expressed their belief that medicinal plants can cure COVID-19 related symptoms and cure infection (Figure 9).

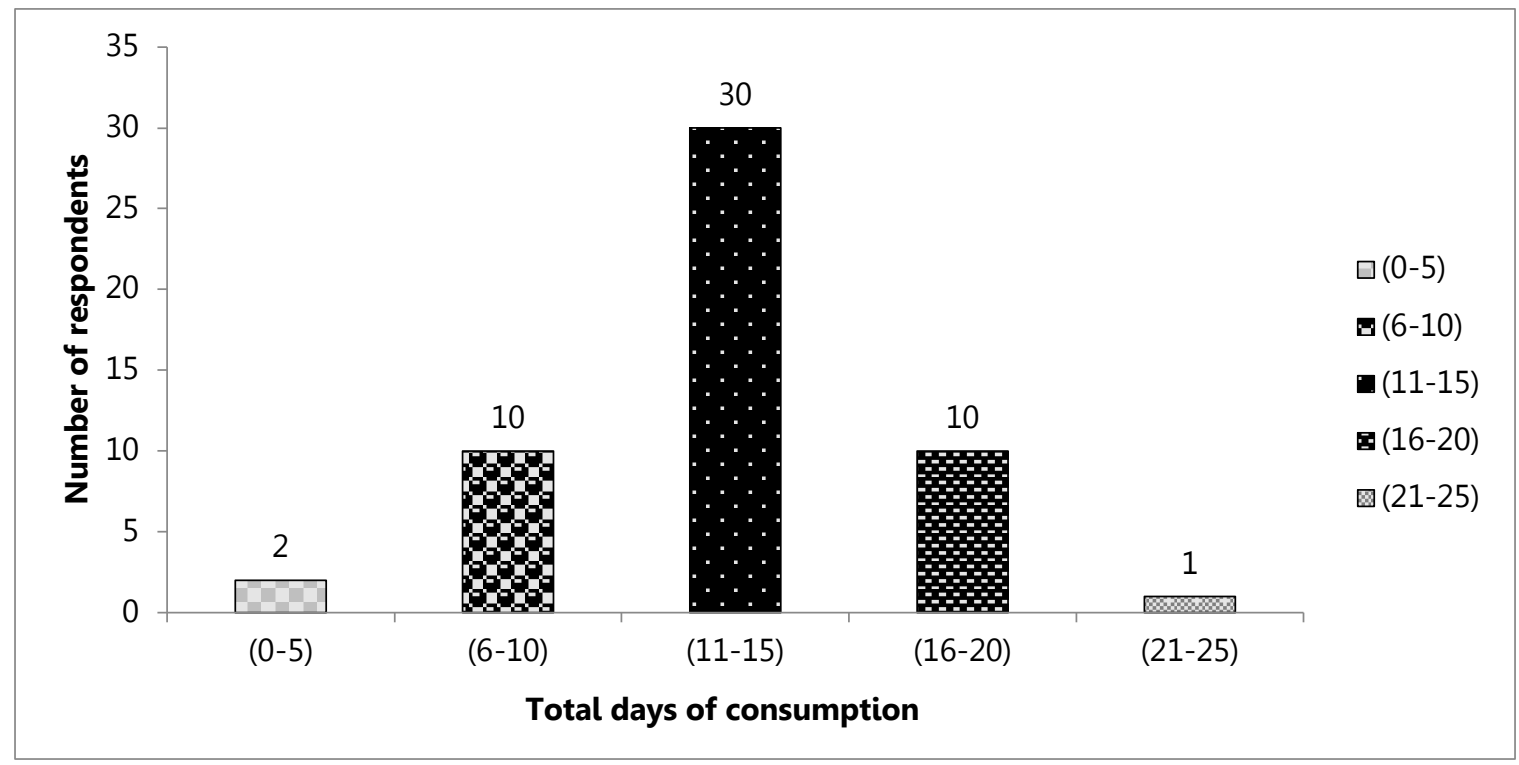

Figure 7. A number of days of consumption of medicinal plants by the 59 COVID-19 survivors. 


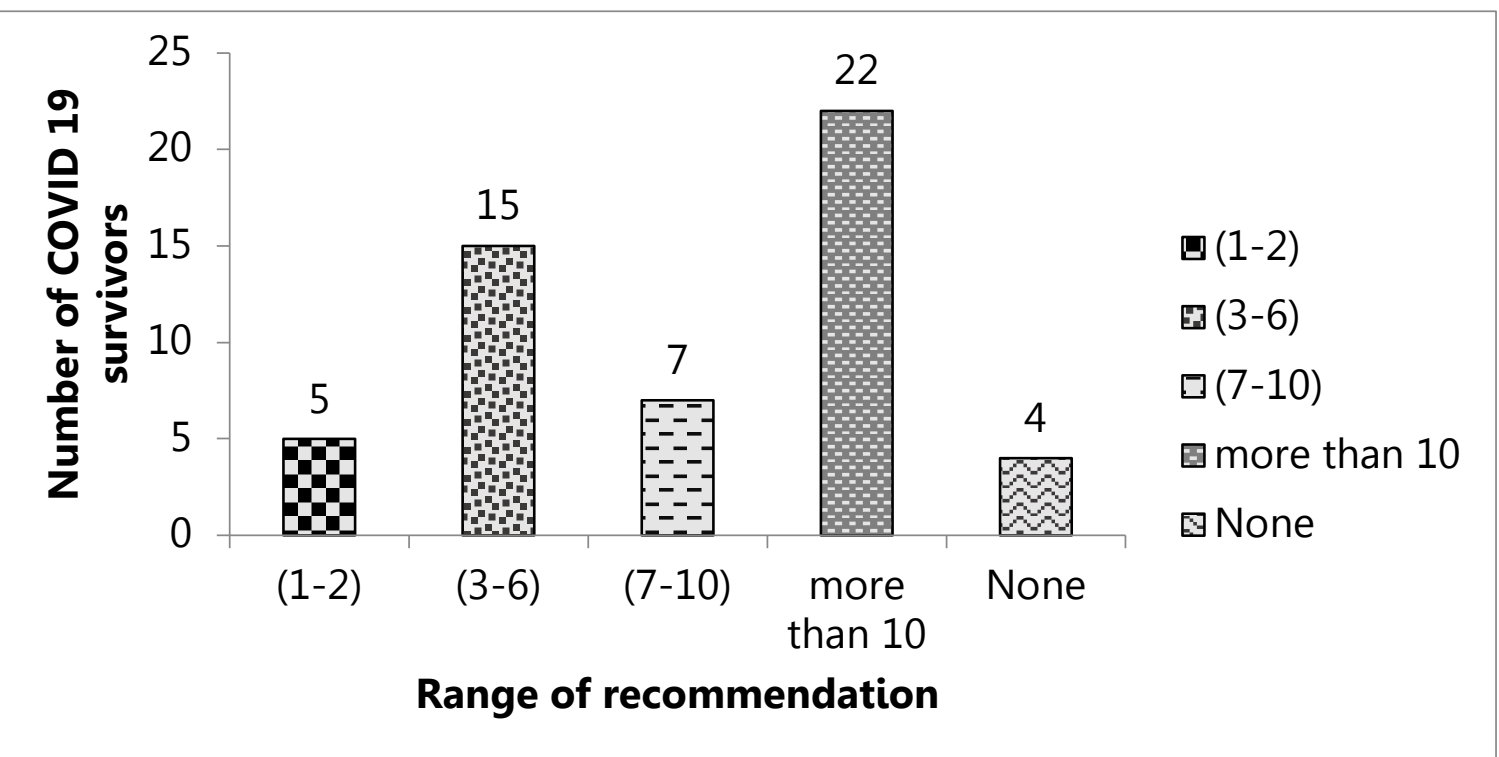

Figure 8. Number of recommended individuals to use medicinal plants by COVID-19 survivors

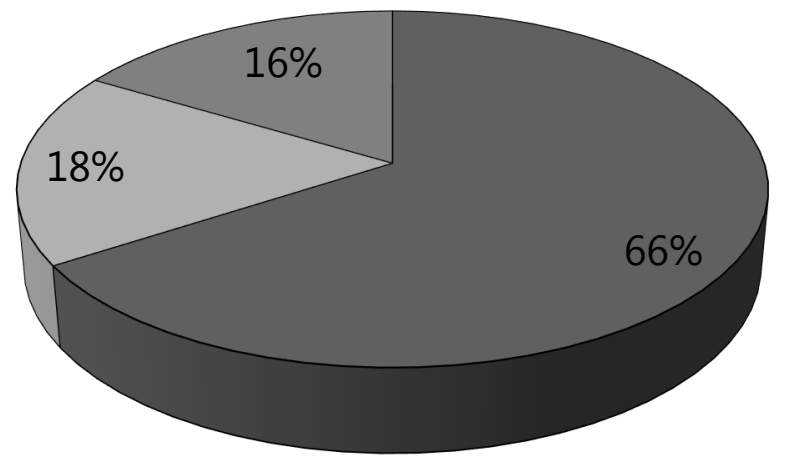

Improve immunity but don't protect from Covid infection

$\square$ Reduce symptoms and cure infection

$\square$ Reduce symptoms but don't cure the infection

Figure 9. Perceived efficacy of medicinal plants.

\section{Relative frequency of citation}

The relative frequency of citations of the top-ranked 15 species among respondents ranged from 0.16 to 0.80 (Figure $10)$. Among them, Curcuma longa (0.80) was the most cited species and Zanthoxylum armatum (0.16) was the least cited species. 


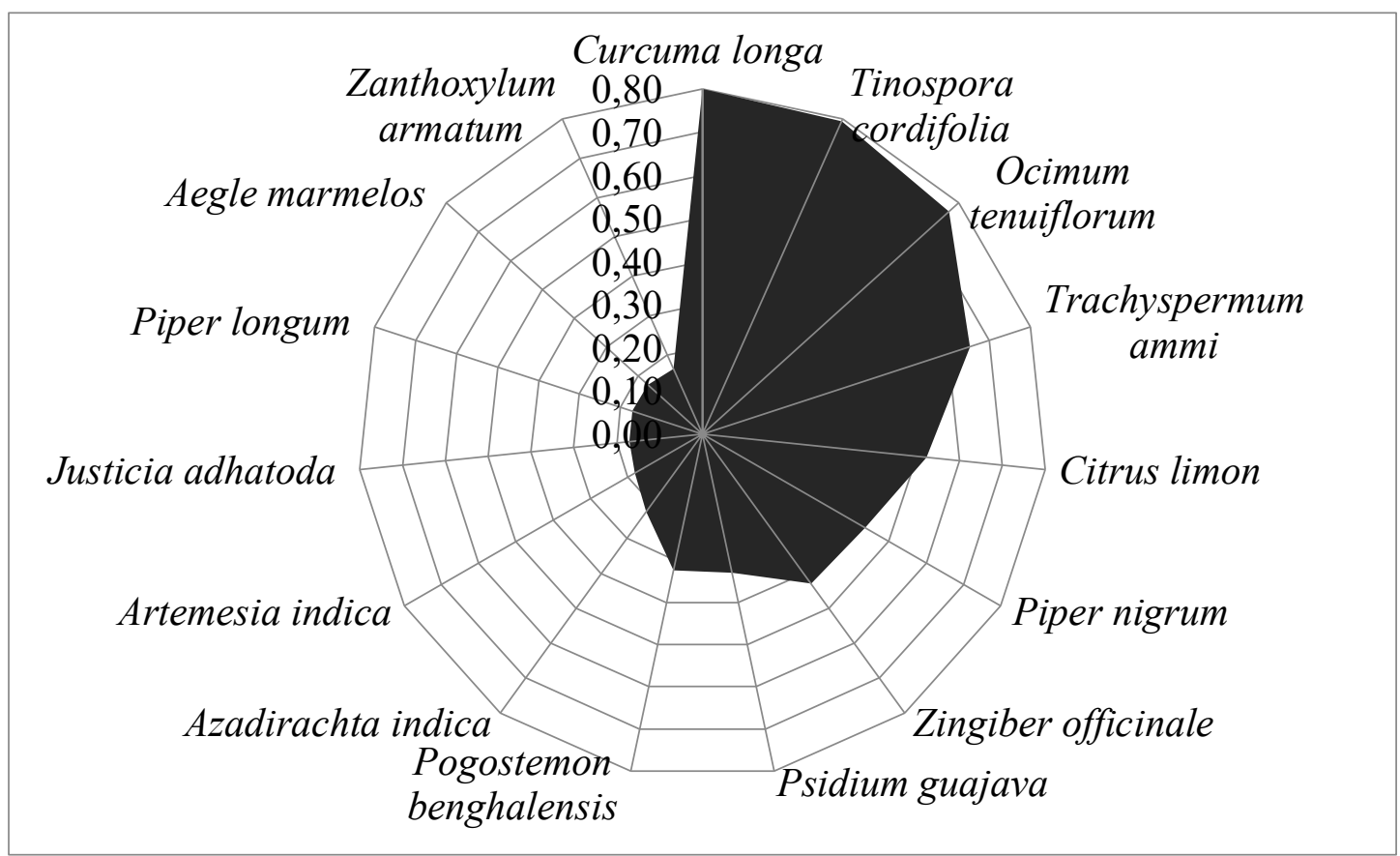

Figure 10. Radar chart showing the relative frequency of citation of the 15 most frequently cited medicinal plant species.

Phytochemical and pharmacological properties of the top $\mathbf{1 5}$ medicinal plants used by the respondents To ensure the efficacy of the medicinal plant in curing COVID-19 symptoms and preventing COVID-19 infection, the top 15 medicinal plant species with the highest relative frequency of citations were selected (Figure 10) and their phytochemical and pharmacological properties were analyzed based on the published pieces of literature. Such analysis showed that all the top 15 medicinal plant species had antiviral and anti-inflammatory properties and 14 of them also had immunomodulatory properties (Table 2). In addition, all top 15 medicinal plant species had been used in past for the treatment of SARS-CoV, Herpes Simplex Virus, Chickungunya Viruses, Zika Virus, Ebola Virus, HIV, SARS-CoV-2, Influenza Virus, Dengue Virus, Hepatitis B Virus, Japanese Encephalitis, and Coxsackieviruses.

Table 2. Phytochemical and pharmacological properties of the 15 most frequently cited medicinal plant species. Scientific names of the species and their respective constituent phytochemicals, pharmacological properties, existing/past antiviral uses, and references are shown.

\begin{tabular}{|c|c|c|c|}
\hline Plant Species & $\begin{array}{l}\text { Constituent } \\
\text { phytochemicals }\end{array}$ & $\begin{array}{l}\text { Pharmacological } \\
\text { properties }\end{array}$ & Existing/past antiviral uses \\
\hline Curcuma longa L. & $\begin{array}{l}\text { Curcumin, } \\
\text { Curcuminoids, } \\
\text { Sesquiterpenes } \\
\text { (Adhikari et al. 2021, } \\
\text { Anand et al. 2021, } \\
\text { Gyawali et al. 2020, } \\
\text { Sharifi-Rad et al. 2020, } \\
\text { Singh et al. 2021) }\end{array}$ & $\begin{array}{l}\text { Antioxidant, Anti- } \\
\text { inflammatory, Anti-cancer, } \\
\text { Antimicrobial, Antiviral, } \\
\text { Immunomodulatory (Anand } \\
\text { et al. 2021, Gyawali et al. } \\
\text { 2020, Sharifi-Rad et al. 2020, } \\
\text { Singh et al. 2021) }\end{array}$ & $\begin{array}{l}\text { SARS-CoV, Herpes simplex } \\
\text { virus, } \\
\text { Chickungunya virus, } \\
\text { Zika virus, } \\
\text { HIV (Gyawali et al. 2020, Singh } \\
\text { et al. 2021) }\end{array}$ \\
\hline $\begin{array}{l}\text { Tinospora } \\
\text { cordifolia (Willd.) } \\
\text { Miers }\end{array}$ & $\begin{array}{l}\text { Alkaloids, Diterpenoid } \\
\text { lactones, } \\
\text { Sesquiterpenoids, } \\
\text { Tinocordifolin } \\
\text { (Ghatpande et al. 2019, } \\
\text { Gyawali et al. 2020, } \\
\text { Tiwari et al. 2018) }\end{array}$ & $\begin{array}{l}\text { Anticancer, Antiviral } \\
\text { Antioxidant, Antidiabetic, } \\
\text { Anti-inflammatory, } \\
\text { Immunomodulatory, } \\
\text { Antimicrobial (Ghatpande et } \\
\text { al. 2019, Gyawali et al. 2020, } \\
\text { Singh et al. 2021, Tiwari et al. } \\
\text { 2018) }\end{array}$ & $\begin{array}{l}\text { SARS-CoV-2, } \\
\text { Chickungunya virus, } \\
\text { HIV } \\
\text { (Gyawali et al. 2020, Singh et } \\
\text { al. 2021) }\end{array}$ \\
\hline
\end{tabular}




\begin{tabular}{|c|c|c|c|}
\hline $\begin{array}{l}\text { Ocimum } \\
\text { tenuiflorum } \mathrm{L} .\end{array}$ & $\begin{array}{l}\text { Phenols, fatty acid, } \\
\text { luteolin, saponins, } \\
\text { tannins, linalool } \\
\text { (Bano et al. 2017, Da } \\
\text { silva et al. 2020, } \\
\text { Gyawali et al. 2020) }\end{array}$ & $\begin{array}{l}\text { Antimicrobial, Antibacterial, } \\
\text { Antiviral, Antifungal, } \\
\text { Antidiabetic, Anticancer, } \\
\text { Anti- inflammatory, } \\
\text { Immunomodulatory (Da } \\
\text { silva et al. 2020, Gulati et al. } \\
\text { 2002, Gyawali et al. 2020, } \\
\text { Kumar et al. 2012, Romeilah } \\
\text { et al. 2010, Singh et al. 2021) }\end{array}$ & $\begin{array}{l}\text { SARS-CoV-2, } \\
\text { H9N2 virus, } \\
\text { Coxsackie virus } \\
\text { (Singh } \text { et al. 2021) }\end{array}$ \\
\hline $\begin{array}{l}\text { Azadirachta indica } \\
\text { A. Juss. }\end{array}$ & $\begin{array}{l}\text { Azadirachtin, Nimbidin, } \\
\text { Nimbin, Nimbolide, } \\
\text { Nimbic acid (Baharek et } \\
\text { al. 2014) }\end{array}$ & $\begin{array}{l}\text { Antimalarial, Antiviral, } \\
\text { Antibacterial, Antifungal, } \\
\text { Anti-inflammatory, } \\
\text { Immunomodulatory } \\
\text { (Baharek et al. 2014, Da silva } \\
\text { et al. 2020, Gulati et al. 2002, } \\
\text { Singh et al. 2021) }\end{array}$ & $\begin{array}{l}\text { SARS-CoV-2, } \\
\text { Influenza virus, } \\
\text { Dengue virus } \\
\text { (Singh et al. 2021) }\end{array}$ \\
\hline $\begin{array}{l}\text { Zingiber officinale } \\
\text { Roscoe }\end{array}$ & $\begin{array}{l}\text { Gingerol, Shogaols, } \\
\text { Paradol, Zingerone } \\
\text { (Gyawali et al. 2020, } \\
\text { Mishra et al. 2012) }\end{array}$ & $\begin{array}{l}\text { Anticancer, Anti- } \\
\text { inflammatory, Antioxidant, } \\
\text { Antimicrobial, } \\
\text { Immunomodulatory, } \\
\text { Antiviral (Da silva et al. 2020, } \\
\text { Gulati et al. 2002, Gyawali et } \\
\text { al. } 2020 \text { Mishra et al. 2012, } \\
\text { Singh et al. 2021) }\end{array}$ & $\begin{array}{l}\text { SARS-CoV-2, } \\
\text { Influenza virus, } \\
\text { Herpes simplex virus, } \\
\text { Chickungunya virus (Singh et } \\
\text { al. 2021) }\end{array}$ \\
\hline $\begin{array}{l}\text { Trachyspermum } \\
\text { ammi (L.) Sprague }\end{array}$ & $\begin{array}{l}\text { Thymol, Thymene, } \\
\text { Limonene, Glycosides } \\
\text { (Kumar \& Khurana } \\
\text { 2018) }\end{array}$ & $\begin{array}{l}\text { Antibacterial, Antiviral, } \\
\text { Antidiarrheal, Antifungal, } \\
\text { Antioxidant, Anti- } \\
\text { inflammatory, } \\
\text { Immunomodulatory (Gupta } \\
\text { et al. 2021, Kumar \& } \\
\text { Khurana 2018) }\end{array}$ & $\begin{array}{l}\text { Japanese encephalitis virus } \\
\text { (Ruwali et al. 2018) }\end{array}$ \\
\hline $\begin{array}{l}\text { Citrus limon (L.) } \\
\text { Osbeck }\end{array}$ & $\begin{array}{l}\text { Flavonoids, Limonoids, } \\
\text { Phenolic acids (Klimek- } \\
\text { szczykutowicz et al. } \\
\text { 2020) }\end{array}$ & $\begin{array}{l}\text { Anticancer, Antioxidant, } \\
\text { Anti-inflammatory, } \\
\text { Antimicrobial, Antiparasitic, } \\
\text { Antifungal, Antiviral, } \\
\text { Immunomodulatory } \\
\text { (Anastasiou \& Buchbauer } \\
\text { 2017, Klimek-szczykutowicz } \\
\text { et al. 2020) }\end{array}$ & $\begin{array}{l}\text { Herpes simplex virus } \\
\text { (Klimek-szczykutowicz et al. } \\
\text { 2020) }\end{array}$ \\
\hline Piper nigrum L. & $\begin{array}{l}\text { Piperine, Piperamine, } \\
\text { Piperamide, Pipericide } \\
\text { (Damanhouri 2014) }\end{array}$ & $\begin{array}{l}\text { Antimicrobial, Antioxidant, } \\
\text { Anticancer, Anti- } \\
\text { inflammatory, } \\
\text { Antidepressant, Antiviral, } \\
\text { Immunomodulatory } \\
\text { (Anastasiou \& Buchbauer } \\
\text { 2017, Damanhouri 2014, } \\
\text { Singh et al. 2021) }\end{array}$ & $\begin{array}{l}\text { Hepatitis } B, \\
\text { Dengue virus, } \\
\text { Ebola virus } \\
\text { (Singh et al. 2021) }\end{array}$ \\
\hline Psidium guajava L. & $\begin{array}{l}\text { Gallic acid, Catechin, } \\
\text { Kaempferol, } \\
\text { Chlorogenic acid, } \\
\text { Quercetin, Myricetin } \\
\text { (Kumar et al. 2021) }\end{array}$ & $\begin{array}{l}\text { Anticancer, Antidiabetic, } \\
\text { Antioxidant, Antimicrobial, } \\
\text { Antidiarrhea, Anti- } \\
\text { inflammatory, } \\
\text { Immunomodulatory, } \\
\text { Antiviral (Mittal \& Gupta } \\
\text { 2010, Sriwilaijaroen et al. } \\
\text { 2012) }\end{array}$ & $\begin{array}{l}\text { Influenza A virus } \\
\text { (Sriwilaijaroen et al. 2012) }\end{array}$ \\
\hline
\end{tabular}




\begin{tabular}{|c|c|c|c|}
\hline Piper longum L. & $\begin{array}{l}\text { Piperine, Methyl } \\
\text { piperine, Sesamin, } \\
\text { Pulvutilol, (Zaveri et al. } \\
\text { 2010) }\end{array}$ & $\begin{array}{l}\text { Antioxidant, Antifungal, } \\
\text { Antimicrobial, Anti- } \\
\text { inflammatory, Anticancer, } \\
\text { Antiviral, } \\
\text { Immunomodulatory (Priya \& } \\
\text { Saravana 2017, Sharma } \\
\text { 2019, Zaveri et al. 2010) }\end{array}$ & $\begin{array}{l}\text { Vesicular stomatitis Indiana } \\
\text { virus, Human para influenza } \\
\text { viruses (Priya \& Saravana 2017) }\end{array}$ \\
\hline $\begin{array}{l}\text { Pogostemon } \\
\text { benghalensis } \\
\text { (Burm. f.) Kuntze }\end{array}$ & $\begin{array}{l}\text { Phenolics, Steroids, } \\
\text { Tannins, Flavonoids, } \\
\text { Terpenoids (Dahiya et } \\
\text { al. 2020) }\end{array}$ & $\begin{array}{l}\text { Antioxidant, Antibacterial, } \\
\text { Antiviral, Antifungal, } \\
\text { Anticancer, Anti- } \\
\text { inflammatory (Dahiya et al. } \\
\text { 2020) }\end{array}$ & $\begin{array}{l}\text { Herpes simplex virus, } \\
\text { Sindbis virus, } \\
\text { Polio virus } \\
\text { (Dahiya et al. 2020) }\end{array}$ \\
\hline $\begin{array}{l}\text { Zanthoxylum } \\
\text { armatum DC. }\end{array}$ & $\begin{array}{l}\text { Berberine, Dictamnine, } \\
\text { limonene, linalool, } \\
\text { Kaempferol (Paul et al. } \\
\text { 2018) }\end{array}$ & $\begin{array}{l}\text { Anti-inflammatory, } \\
\text { Antibacterial, Antifungal, } \\
\text { Antioxidant, Antiviral, } \\
\text { Immunomodulatory (Alam et } \\
\text { al. 2020, Bharti \& Bhusan } \\
\text { 2017, Gyawali et al. 2020, } \\
\text { Paul et al. 2018) }\end{array}$ & $\begin{array}{l}\text { Herpes simplex virus, } \\
\text { Influenza virus } \\
\text { (Gyawali et al. 2020) }\end{array}$ \\
\hline $\begin{array}{l}\text { Aegle marmelos } \\
\text { (L.) Correa }\end{array}$ & $\begin{array}{l}\text { Alkaloids, Coumarins, } \\
\text { Flavonoids, Marmelide, } \\
\text { Tannins (Manandhar et } \\
\text { al. 2018) }\end{array}$ & $\begin{array}{l}\text { Antioxidant, Antidiabetic, } \\
\text { Antimicrobial, Antiviral, } \\
\text { Anticancer, Anti- } \\
\text { inflammatory, } \\
\text { Immunomodulatory (Badam } \\
\text { et al. 2002, Govinda \& } \\
\text { Asdaq 2011, Manandhar et } \\
\text { al. 2018) }\end{array}$ & $\begin{array}{l}\text { Human coxsackieviruses B1-B6 } \\
\text { (Badam et al. 2002) }\end{array}$ \\
\hline Justicia adhatoda L. & $\begin{array}{l}\text { Adhatodine, Anisotine, } \\
\text { Vasicoline, } \\
\text { Vasicolinone (Kaur et } \\
\text { al. 2013) }\end{array}$ & $\begin{array}{l}\text { Anti-inflammatory, } \\
\text { Antimicrobial, Antibacterial, } \\
\text { Antioxidant, Antiviral, } \\
\text { Immunomodulatory (Kaur et } \\
\text { al. 2013, Singh et al. 2017) }\end{array}$ & $\begin{array}{l}\text { Herpes simplex virus-1 and } 2 \\
\text { (Singh et al. 2017) }\end{array}$ \\
\hline $\begin{array}{l}\text { Artemesia indica } \\
\text { Willd. }\end{array}$ & $\begin{array}{l}\text { Flavonoids, Coumarins, } \\
\text { Sesquiterpene, } \\
\text { Lactones, Alkaloids } \\
\text { (Bisht et al. 2021) }\end{array}$ & $\begin{array}{l}\text { Antioxidant, Antimicrobial, } \\
\text { Antifungal, Antibacterial, } \\
\text { Anticancer, } \\
\text { Anti-inflammatory, Antiviral, } \\
\text { Immunomodulatory (Bisht et } \\
\text { al. 2021, Kshirsagar \& Rao } \\
\text { 2021, Ruwali et al. 2018) }\end{array}$ & $\begin{array}{l}\text { Hepatitis B and C viruses, HIV- } \\
\text { 1, Influenza virus A (Kshirsagar } \\
\text { \& Rao } 2021\end{array}$ \\
\hline
\end{tabular}

\section{Discussion}

COVID-19 infection is highly contagious (Gasmi et al. 2020), and there is no effective antiviral treatment to combat the disease until date (Singh et al. 2021). Few vaccines have been approved for emergency use and have been claimed to provide a high degree of protection against getting seriously ill and dying from the disease ( $L i$ et al. 2021). However, not everyone has access to those vaccines (Forni \& Mantovani 2021). This has driven increased interest in exploring alternatives of medicinal plant-based therapeutics globally. In this context, our study highlights the importance and perceived efficacy of medicinal plants in the treatment and prevention of COVID-19.

The use of phytomedicine has been an integral aspect of the traditional health care system for thousands of years (Ghoran et al. 2021). The usage of medicinal plants among patients has gained popularity, and considerable literature has reported on their usage in the case of new infectious diseases such as SARS and MERS (Khadka et al. 2021). Our study recorded 41 species of medicinal plants belonging to 24 different families and 37 genera being used extensively by the inhabitants of Buddhabhumi Municipality for the treatment and prevention of COVID-19. The majority of the species are similar to the species recorded by analogous but extensive studies from Nepal (Khadka et al. 2021) and other countries such as India (Srivastava et al. 2020), China (Shahrajabian et al. 2020, Xu \& Zhang 2020), Bangladesh (Bhuiyan et al. 2020), Morocco (Alami et al. 2020), Peru (Villena-Tejada et al. 2021), Brazil, Jamaica, Bolivia, Romania, Belarus, Lithuania, Poland, Georgia, Turkey, Pakistan, Cambodia, and South Africa (Pieroni 
et al. 2020). However, the recording of 41 species of medicinal plants from a municipality of Nepal is much higher compared to 60 species recorded by Khadka et al. (2021), from across the country. This hints towards the increasing popularity of medicinal plants among infected and non-infected people.

Our study documented species such as Curcuma longa, Coriandrum sativum, Allium sativum, and Cuminum cyminum that are recommended for cooking to combat COVID-19 and species like Ocimum tenuiflorum, Zingiber officinale, Piper nigrum, and Cinnamomum tamala that are recommended for decoction (Gupta et al. 2021). Additionally, species like Tinospora cordifolia, Curcuma longa, Zingiber officinale, Ocimum tenuiflorum, Zanthoxylum armatum, Phyllanthus emblica, Allium sativum, Withania somnifera, and Piper cubeba that our study documented have already been recommended as remedies for COVID-19 by Gyawali et al. (2020). Medicinal herbs have always been part of Nepalese home gardens (Pokhrel 2015) and are traditionally used in many therapeutic practices (Babich et al. 2020). Our study confirms that herbs are among the most frequently used medicinal plants and most of the medicinal plants that are being used are sourced from the respondents' home gardens. Nevertheless, a minority of the respondents reported that they are getting medicinal plants from the nearest forest areas. A disordered accumulation of medicinal plants, especially underground parts, and whole plants could pose a threat to nature conservation (Hussain et al. 2013). Leaves are storehouses of active secondary metabolites (Ghorbani 2005), and our study has confirmed that leaves are among the most highly used plant parts.

Many of the medicinal plant species documented by our study are already part of the list of alternative medicines to boost the immunity power included in the Ayurveda and Alternative Medicine Guidelines of Preventive Measures and Management Protocol for COVID-19 in Nepal published by the Department of Ayurveda \& Alternative Medicine (DAAM), Ministry of Health \& Population, Nepal (MOHP 2020). The majority of the respondents expressed their belief that medicinal plants can enhance their immunity against viruses is in line with the aforementioned guidelines of the DAAM.

The eccentricity of the population has increased the use of medicinal plants with antiviral and anti-inflammatory properties to enhance the immune system or cure respiratory diseases (Maldonado et al. 2020). Research on ethnopharmacology is full of possibilities, yet challenging, since a single plant may contain a wide range of phytochemicals (Lim et al. 2021). The phytochemical and pharmacological screening of the top 15 medicinal plant species documented in this study showed that all the top 15 medicinal plant species had antiviral and antiinflammatory properties, whereas 14 of them also had immunomodulatory properties. Additionally, the 15 most frequently cited species have been reported to be used or be used for the SARS-CoV, Herpes Simplex Virus, Chickungunya Viruses, Zika Virus, Ebola Virus, HIV, SARS-CoV-2, Influenza Virus, Dengue Virus, Hepatitis B Virus, Japanese Encephalitis, and Coxsackieviruses, etc. Evidence from the research conducted on SARS-COV and COVID19 has shown that a fragile immune system is one of the major contributing factors for COVID complications (Curbelo et al. 2017; Promptetchara et al. 2020, Taghizadeh-Hesary \& Akbari 2020). Therefore, improving the immune system could avoid COVID-19 infection and complications (Fedoung et al. 2021). Phytochemicals such as curcumin, gingerol, zingerol, flavonoids, catechins, quercetin, kaempferol, alkaloids, saponins, and tannins present in the 15 most frequently cited medicinal plants were proven to be antiviral bioactive compounds by the studies of Adhikari et al. (2021), Anand et al. (2021), Ghoran et al. (2021). Moreover, global studies have demonstrated that many medicinal plants have antiviral activity against coronavirus and their primary mechanism seems to be through inhibition of viral replication (Jassim \& Naji 2003). In China, traditional Chinese medicinal herbs have been used for the effective treatment of SARS. Given their phytochemical and pharmacological properties, the 15 most frequently cited medicinal plant species could be the best candidates in herbal drug formulations for the treatment of COVID19 in the future. The significance of medicinal plants to counteract SARS-CoV patented from 2008- 2013 for their anti-SARS-CoV activities is reflected in a review article by Kumar et al. (2013). Provided the Government of Nepal and the Government of India (Gol) have formally declared that the Traditional System of Medicine (TSM) should be used in conjunction with allopathic medicine in the treatment of COVID-19 patients (Pandit \& Singh 2020).

\section{Conclusion}

The study found that 41 medicinal plant species from 24 different families and 37 genera are used. Enthralling, all of the 15 most frequently cited medicinal plants were reported to have both antiviral and anti-inflammatory properties and 14 of them were reported to have immunomodulatory properties. They were reported to be effective against various fatal viruses and could be the best candidates in herbal drug formulations for the treatment of COVID-19 in the future. There is a wide range of potential uses of traditional medicines in these challenging times due to their long history of use in the community, ancient references, and scientific evidence about their safety and efficacy. In conclusion, our study suggests that medicinal plants are effective against viral infections such as COVID- 
19 and encourages us to get closer to the natural healing process. Although there are still many challenges and questions unanswered, the recovery of COVID-19 infected individuals by using medicinal plants offers a hope that this pandemic can be halted with herbal drug formulations. Regarding using medicinal plants, sources of information are crucial. It is not advisable to rely on informal sources and unsubstantiated efficacy claims on social media. However, instead of relying on formal and authentic sources such as local and national health authorities, people are using medicinal plants, knowingly or unknowingly for the treatment of the miscellaneous symptoms of COVID-19 preventative measure against COVID-19 infection. Therefore, our study appeals to the concerned authorities such as the Ministry of Health (MoHP) and Population, pharmaceutical companies, pharmacologists, ethnobotanists, and phytochemists to take the potential of medicinal plants seriously and immediately initiate further research on these antiviral, anti-inflammatory and immunomodulatory medicinal plants so that we could pave way for the formulation of novel drugs for the effective treatment of COVID-19.

\section{Declarations}

List of abbreviations: Habit: $\mathrm{H}=$ Herb, $\mathrm{S}=$ Shrub, $\mathrm{C}=$ Climber, $\mathrm{T}=$ Tree

Parts used: $\mathrm{L}=$ Leaf, $\mathrm{R}=$ Rhizome, $\mathrm{Se}=\mathrm{Seed}, \mathrm{F}=$ Fruits, $\mathrm{St}=$ Stem, $\mathrm{Fl}=$ Flower, $\mathrm{W}=$ Whole plant, $\mathrm{B}=\mathrm{Bark}, \mathrm{Bu}=\mathrm{Bulb}$, Rt $=$ Root

Ethics approval and consent to participate: We strictly followed the ethical guidelines of the International Society of Ethnobiology (http://www.ethnobiology.net/). The consent message was written to each recipient and placed clearly on the top of the form.

Consent for publication: Not applicable

Availability of data and materials: The data set generated for the current study is available upon request.

Competing Interests: The authors declare that they have no conflict of interest.

Funding: This research did not receive any specific grants from funding agencies in the public, commercial, or notfor-profit sectors.

Authors' contributions: The authors were involved in concept development and defining intellectual content. VTC conducted the literature research, data collection and prepared a manuscript draft. PJ analyzed the data collected, reviewed, and edited the manuscript. SKM conducted editing, proofreading, and reviewing the manuscript for finalization. The published version of the manuscript has been read and approved by all authors.

\section{Acknowledgments}

We are highly grateful to all respondents who took part in the survey, cooperated during phone and direct interviews. We would like to acknowledge Mr. Resham GC for helping study area map preparation, Ms. Suruchi Bhattarai for proofreading. We would also like to thank the Municipal office for providing relevant records.

\section{Literatures Cited}

Adhikari B, Marasini BP, Rayamajhee B, Bhattarai BR, Lamichhane G, Khadayat K, Adhikari A, Khanal S, Parajuli N. 2021. Potential roles of medicinal plants for the treatment of viral diseases focusing on COVID-19: A review. Phytotherapy Research 35(3):1298-1312.

Adhikari M, Thapa R, Kunwar RM, Devkota HP, Poudel P. 2019. Ethnomedicinal Uses of Plant Resources in the Machhapuchree Rural Municipality of Kaski District, Nepal. Medicines 6(2):69.

Alagaili AN, Briese T, Mishra N, Kapoor V, Sameroff SC, De Wit E, Munster V, Hensley LE, Zalmout IS, Kapoor A. 2014. Middle east respiratory syndrome coronavirus infection in dromedary camels in Saudi Arabia. mBio 5: e00884-14.

Alam F, Din KM, Rasheed R, Sadiq A, Jan MS, Minhas AM, Khan A. 2020. Phytochemical investigation, antiinflammatory, antipyretic and antinociceptive activities of Zanthoxylum armatum DC extracts-in vivo and in vitro experiments. Heliyon 6(11): e05571.

Alami A, Fattah A, Chait A. 2020. Medicinal plants used for the prevention purposes during the covid-19 pandemic in Morocco. Journal of Analalytical Scientce and Applied Biotechnology 2(1):4-11.

Anand AV, Bala muralikrishnan B, Kaviya M, Bharathi K, Parithathvi A, Arun M, Senthilkumar N, Velayutha prabhu S, Saradhadevi M, Al-Dhabi NA, Arasu MV. 2021. Medicinal plants, phytochemicals, and herbs to combat viral pathogens Including SARS-CoV-2. Molecules 26(6):1775.

Anastasiou C, Buchbauer G. 2017. Essential oils as immunomodulators: some examples. Open Chemistry 15(1):352370. 
Babich O, Sukhikh S, Prosekov A, Asyakina L, Ivanova S. 2020. Medicinal Plants to Strengthen Immunity during a Pandemic. Pharmaceuticals 13(10):313.

Badam L, Bedekar S, Sonavane KB, Joshi SP. 2002. In vitro antiviral activity of bael (Aegle marmelos Corr.) upon. Journal of Communicable Diseases 34(2):88-99.

Baharak BS, Mansoor O, Reza NM, Farhad HA, Sepideh KJ, Mehdi S, Mohammad S. 2014. Effect of explants, salts concentration medium and hormone treatments on Taxus baccata in vitro culture. International Journal of Bioscience 5:1-9.

Bano N, Ahmed A, Tanveer M, Khan GM, Ansari MT. 2017. Pharmacological evaluation of Ocimum sanctum. Journal of Bioequivalence and Bioavailability 9(3):387-492.

Benarba B, Pandiella A. 2020. Medicinal Plants as Sources of Active Molecules Against COVID-19. Frontiers in Pharmacology 11.

Bharti S, Bhushan B. 2017. Phytochemical and Pharmacological Activities of Zanthoxylum armatum. Research Journal of Pharmaceutical, Biological and Chemical Sciences 6(1403):1403-1409.

Bhuiyan FR, Howlader S, Raihan T, Hasan M. 2020. Plant metabolites: possibility of natural therapeutics against the COVID-19 pandemic. Frontiers in Medicine (Lausanne) 7:444.

Bisht D, Kumar D, Kumar D, Dua K, Chellappan DK. 2021. Phytochemistry and pharmacological activity of the genus artemisia. Archives of Pharmacal Research 24:1-36.

Bousquet J, Czarlewski W, Zuberbier T, Mullol J, Blain H, Cristol JP, De La Torre R, Le Moing V, Lozano NP, Bed brook A \& Agache I. 2021. Spices to Control COVID-19 Symptoms: Yes, but Not Only.... International Archives of Allergy and Immunology 182(6):489-495.

Central Bureau of Statistics Nepal. Statistical Pocket Book Nepal 2018.2018. http://www.saarcstat.org/content/statistical-officer-central-bureau-statistics-thapathali-kathmandu-nepal. (Accessed 1/6/2021).

Curbelo J, Luquero Bueno S, Galván-Román JM, Ortega-Gómez M, Rajas O, Fernández-Jiménez G, Vega-Piris L, Rodríguez-Salvanes F, Arnalich B, Díaz A, Costa R. 2017. Inflammation biomarkers in blood as mortality predictors in community-acquired pneumonia admitted patients: Importance of comparison with neutrophil count percentage or neutrophil-lymphocyte ratio. PloS one 12(3): e0173947.

Da Silva Antonio A, Wiedemann LS, Veiga-Junior VF. 2020. Natural products' role against COVID-19. RSC Advances 10(39):279-293.

Dahiya S, Batish DR, Singh HP. 2020. Ethnobotanical, phytochemical and pharmacological aspects of Bengal Pogostemon (Pogostemonbenghalensis). Journal of Herbmed Pharmacology 29(4):318-27.

Damanhouri Z, Ahmad A. 2014. A review on therapeutic potential of black pepper (Piper nigrum L.): the king of spices. Journal of Medicinal and Aromatic Plants 3(3):161.

De Wit E, Van Doremalen N, Falzarano D, Munster V. 2016. SARS and MERS: Recent insights into emerging coronaviruses. National Reviuew of Genetice 14: 523-534.

Fedoung EF, Biwole AB, Biyegue CF, Tounkam MN, Ntonga PA, Nguiamba VP, Essono DM, Funwi PF, Tonga C, Nguenang GM \& Kemeuze V. 2021. A review of Cameroonian medicinal plants with potentials for the management of the COVID-19 pandemic. Advances in Traditional Medicine 26:1-26.

Forni G, Mantovani A. 2021. COVID-19 vaccines: where we stand and challenges ahead. Cell Death \& Differentiation 28(2):626-39.

Gasmi A, Noor S, Tippairote T, Dadar M, Menzel A, Bjørklund G. 2020. Individual risk management strategy and potential therapeutic options for the COVID-19 pandemic. Clinical Immunology 108:409.

Ghatpande NS, Misar AV, Wag hole RJ, Jadhav SH, Kulkarni PP. 2019. Tinospora cordifolia protects against inflammation associated anemia by modulating inflammatory cytokines and hepcidin expression in male Wistar rats. Scientific reports $9(1): 1-11$. 
Ghoran SH, El-Shazly M, Sekeroglu N, Kijjoa A. 2021. Natural Products from Medicinal Plants with Anti-Human Coronavirus Activities. Molecules 26(6):1754.

Ghorbani A. 2005. Studies on pharmaceutical ethnobotany in the region of Turkmen Sahra, north of Iran: (Part 1): General results. J Ethnopharmacol 102(1):58-68.

Govinda HV, Asdaq SM. 2011. Immunomodulatory potential of methanol extract of Aegle marmelos in animals. Indian Journal of Pharmaceutical Sciences 73(2):235.

Gulati K, Ray A, Debnath PK, Bhattacharya SK. 2002. Immunomodulatory Indian medicinal plants. Journal of Natural Remedies 2(2):121-131.

Gupta PK, Sone wane K, Rajan M, Patil NJ, Agrawal T, Banerjee ER, Chauhan NS, Kumar A. 2021. Scientific rationale of Indian AYUSH Ministry advisory for COVID-19 prevention, prophylaxis, and immunomodulation. Advances in Traditional Medicine 1-25.

Gyawali R, Paudel PN, Basyal D, Setzer WN, Lamichhane S, Paudel MK, Gyawali S, Khanal P. 2020. A Review on Ayurvedic Medicinal Herbs as Remedial Perspective for COVID-19. Journal of Karnali Academy of Health Sciences 26:3.

Health Office Kapilvastu. Ditrict Health office kapilvastu. 2021. http://hokapilvastu.lumbini.gov.np/.5 (Accessed 16/4/2021).

Hossain M, Paul D, Arfan Ali M, Nurul Huda M, Sarowar Alam M, Mahmood S, Hamooh BT. 2020. The Perspectives of Medicinal Plants for COVID-19 Treatment: A Review. Journal of Agricultural Science \& Engineering Innovation 1(2): 10-17.

Hussain A, Abbasi M, Hussain N, Majid S. 2013. A survey of important indigenous medicinal plants of district Bhimber Azad Jammu \& Kashmir, Pakistan. International Journal of Advanced Research 1:635-644.

International Society of Ethnobiology. 2006. International Society of Ethnobiology Code of Ethics (with 2008 additions). http://ethnobiology.net/ code-of-ethics/

Jassim SA, Naji MA. 2003. Novel antiviral agents: a medicinal plant perspective. Journal of Applied Microbiology 95(3):412-427.

Kaur R, Ruhil S, Balhara M, Dhankhar S, Chhillar AK. 2013. A review on Justicia adhatoda. A potential source of natural medicine. African Journal of Plant Science 5(11):620-627.

Klimek-Szczykutowicz M, Szopa A, Ekiert H. 2020. Citrus limon (Lemon) phenomenon-a review of the chemistry, pharmacological properties, applications in the modern pharmaceutical, food, and cosmetics industries, and biotechnological studies. Plants 9(1):119.

Kshirsagar SG, Rao RV. 2021. Antiviral and immunomodulation effects of Artemisia. Medicina 57(3):217.

Kumar M, Tomar M, Amarowicz R, Saurabh V, Nair MS, Maheshwari C, Sasi M, Prajapati U, Hasan M, Singh S \& Changan S. 2021. Guava (Psidium guajava L.) leaves: Nutritional composition, phytochemical profile, and healthpromoting bioactivities. Foods 10(4):752.

Kumar N, Khurana S. 2018. Phytochemicals and Bioactive Potential of Trachyspermum ammi L. Pharm Lett 10(8):4856.

Kumar PK, Kumar MR, Kavitha K, Singh J, Khan R. 2012. Pharmacological actions of Ocimum sanctum-review article. International Journal of Advances in Pharmacy, Biology and Chemistry 1(3):277-288.

Kumar V, Jung YS, Liang PH. 2013. Anti-SARS coronavirus agents: A patent review (2008-Present). Expert Opinions on Therapeutic Patents 23:1337-1348.

Kunwar RM, Shrestha KP, Bussmann RW. 2010. Traditional herbal medicine in far-west Nepal: a pharmacological appraisal. Journal of Ethnobiology and Ethnomedicine 6:35.

Lake MA. 2020. What we know so far: COVID-19 current clinical knowledge and research. Clinical Medicine 20(2):124.

Lee N, Hui DS, Wu A, Chan PKS, Cameron P, Joynt G, Ahuja AT, Yung MY, Leung C, To K. 2003. A major outbreak of severe acute respiratory syndrome in Hong Kong. New England Journalof Medicine 348: 1986-1994. 
Li Y, Liu X, Guo L, Li J, Zhong D, Zhang Y, Clarke M, Jin R. 2020. Traditional Chinese herbal medicine for treating novel coronavirus (COVID-19) pneumonia: Protocol for a systematic review and meta-Analysis. Systematic Reviews 9(1):1-7.

Li Y, Tenchov R, Smoot J, Liu C, Watkins S, Zhou Q. 2021. A Comprehensive Review of the Global Efforts on COVID19 Vaccine Development. ACS Central Science 7(4):512-33.

Lim XY, Teh BP, Tan TY. 2021. Medicinal Plants in COVID-19: Potential and Limitations. Frontiers in Pharmacology 12:355.

Maldonado C, Paniagua-Zambrana N, Bussmann RW, Zenteno-Ruiz FS, Fuentes AF. 2020. The importance of medicinal plants, their taxonomy and the search for a cure to the disease caused by the coronavirus (COVID-19). Ecology in Bolivia 55 (1): 1-5.

Manandhar B, Paudel KR, Sharma B, Karki R. 2018. Phytochemical profile and pharmacological activity of Aegle marmelos Linn. Journal of Integrative Medicine 16(3):153-63.

Memish ZA, Mishra N, Olival KJ, Fagbo S, Kapoor V, Epstein JH, Al Hakeem R, Durosinloun A, Al Asmari M \& Islam A. 2013. Middle East respiratory syndrome coronavirus in bats, Saudi Arabia. Emerging Infectious Diseases 19:18191823.

Ministry of Health and Population. COVID19-Dashboard. 2021. https://covid19.mohp.gov.np. (Accessed 22/8/2021).

Mishra RK, Kumar A, Kumar A. 2012. Pharmacological activity of Zingiber officinale. International Journal of Pharmaceutical and Chemical Sciences 1(3):173-180.

Mittal P, Gupta V, Kaur G, Garg A, Singh A. 2010. Phytochemistry and pharmacological activities of Psidium guajava. International Journal of Pharmaceutical Science Research 1(9):9-19.

Mohammed AA, Al-Aaragi AN, Merzah MA. 2018. Knowledge, attitude, and practice regarding diabetic mellitus among a sample of students at technical institute of Karbala. Medical Journal of Babylon 15(2):164.

MoHP. 2020. Ayurveda and Alternative Medicine Guidelines of Preventive Measures and Management Protocol for COVID 19 in Nepal. In: Department of Ayurveda \& Alternative Medicine, Nardevi NAMC, Ayurveda Campus \& Teaching Hospital K, editors.

Pandey A, Khan MK, Hamurcu M, Gezgin S. 2020. Natural Plant Products: A Less Focused Aspect for the COVID-19 Viral Outbreak. Frontiers in Plant Science 11.

Pandit RD, Singh RK. 2020. COVID-19 Ayurveda treatment protocol of governments of Nepal and India: a review and perspective. Applied Science and Technology Annals 1(1):72-80.

Paul A, Kumar A, Singh G, Choudhary A. 2018. Medicinal, pharmaceutical and pharmacological properties of Zanthoxylum armatum. A Review. Journal of Pharmacogoscy and Phytochemistry 7(4):892-900.

Peiris J, Lai S, Poon LL, Guan Y, Yam L, Lim W, Nicholls JM, Yee W, Yan W, Cheung M. 2003. Coronavirus as a possible cause of severe acute respiratory syndrome. Lancet 361: 1319-1325.

Pokhrel CP. 2015. Assessment of plant diversity in home gardens of three ecological zones of Nepal. Ecoprint. An International Journal of Ecology 22:63-74.

Priya NC, Saravana Kumari P. 2017. Antiviral activities and cytotoxicity assay of seed extracts of Piper longum and Piper nigrum on human cell lines. International Journal of Pharmaceutical Sciences Review and Research 44(1):197202.

Prompetchara E, Ketloy C, Palaga T. 2020. Immune responses in COVID-19 and potential vaccines: Lessons learned from SARS and MERS epidemic. Asian Pacific Journal of Allergy and Immunology 38(1):1-9.

Reuters Nepal. the latest coronavirus counts, charts and maps. 2021. https://graphics.reuters.com/worldcoronavirus-tracker-and-maps/countries-and-territories/nepal/. (Accessed 13/6/2021).

Romeilah RM, Fayed SA, Mahmoud GI. 2010. Chemical compositions, antiviral and antioxidant activities of seven essential oils. Journal of Applied Science Research 6(1):50-62. 
Ruwali P, Ambwani TK, Gautam P. 2018. In vitro immunomodulatory potential of Artemisia indica Willd. in chicken lymphocytes. Veterinary world 11(1):80.

Saad-Roy CM, Wagner CE, Baker RE, Morris SE, Farrar J, Graham AL, Levin SA, Mina MJ, Metcalf CJ, Grenfell BT. 2020. Immune life history, vaccination, and the dynamics of SARS-CoV-2 over the next 5 years. Science 370(6518):811818.

Salathé M, Althaus CL, Neher R, Stringhini S, Hodcroft E, Fellay J, Zwahlen M, Senti G, Battegay M, Wilder-Smith A, Eckerle I. 2020. COVID-19 epidemic in Switzerland: on the importance of testing, contact tracing and isolation. Swiss medical weekly 19:150.

Shahrajabian MH, Sun W, Shen H, Cheng Q. 2020. Chinese herbal medicine for SARS and SARS-CoV-2 treatment and prevention, encouraging using herbal medicine for COVID-19 outbreak. Acta Agriculturae Scandinaviae Sect BSoil Plant Science 70(5):437-443

Sharifi-Rad J, Rayess YE, Rizk AA, Sadaka C, Zgheib R, Zam W, Sestito S, Rapposelli S, Neffe-Skocińska K, Zielińska D, Salehi B. 2020. Turmeric and its major compound curcumin on health: bioactive effects and safety profiles for food, pharmaceutical, biotechnological and medicinal applications. Frontiers in Pharmacology 1-23.

Sharma P, Kumar P, Sharma R, Gupta G, Chaudhary A. 2017. Immunomodulators: Role of medicinal plants in immune system. National Journal of Physiology, Pharmacy and Pharmacology 7(6):552.

Sharma R. 2019. Viral Diseases and Antiviral Activity of Some Medicinal Plants with Special Reference to Ajmer. Journal of Antivirals and Antiretrovirals 11:183.

Shereen MA, Khan S, Kazmi A, Bashir N, Siddique R. 2020. COVID-19 infection: Origin, transmission, and characteristics of human coronaviruses. Journal of Advanced Research 24:91-108.

Singh NA, Kumar P, Kumar N. 2021. Spices and herbs: Potential antiviral preventives and immunity boosters during COVID-19. Phytotherapy Research 1-13

Singh SK, Patel JR, Dangi A, Bachle D, Kataria RK. 2017. A complete over review on Adhatoda vasica a traditional traditional medicinal plant Medicinal Plants 5(1):175-180.

Srivastava A, Chaurasia J, Khan R, Dhand C, Verma S. 2020. Role of medicinal plants of traditional use in recuperating devastating COVID-19 situation. Medicinal and Aromatic Plants 9(359):2167-0412

Sriwilaijaroen N, Fukumoto S, Kumagai K, Hiramatsu H, Odagiri T, Tashiro M, Suzuki Y. 2012. Antiviral effects of Psidium guajava Linn. (guava) tea on the growth of clinical isolated H1N1 viruses: Its role in viral hemagglutination and neuraminidase inhibition. Antiviral > Research 94(2):139-146.

Taghizadeh-Hesary F, Akbari H. 2020. The powerful immune system against powerful COVID-19: a hypothesis. Medical hypotheses.

Tardío J, Pardo-de-Santayana M. 2008. Cultural importance indices: a comparative analysis based on the useful wild plants of Southern Cantabria (Northern Spain). Economic Botany 62(1):24-39.

Tiwari P, Nayak P, Prusty SK, Sahu PK. 2018. Phytochemistry and pharmacology of Tinospora cordifolia. A review. Systematic Reviews in Pharmacy 9(1):70-78.

Vandebroek I, Pieroni A, Stepp JR, Hanazaki N, Ladio A, Alves RR, Picking D, Delgoda R, Maroyi A, van Andel T, Quave CL. 2020. Reshaping the future of ethnobiology research after the COVID-19 pandemic. Nature Plants 6(7):723-730.

Villena-Tejada M, Vera-Ferchau I, Cardona-Rivero A, Zamalloa-Cornejo R, Quispe-Florez M, Frisancho-Triveño Z, Abarca-Melendez RC, Alvarez-Sucari SG, Mejia CR, Yañez JA. 2021. Use of medicinal plants for COVID-19 prevention and respiratory symptom treatment during the pandemic in Cusco, Peru: A cross-sectional survey. medRxiv.

Wang D, Huang J, Yeung AW, Tzvetkov NT, Horbańczuk JO, Willschke H, Gai Z, Atanasov AG. 2020. The significance of natural product derivatives and traditional medicine for COVID-19. Processes 8(8):937.

WHO. Coronavirus disease (COVID-19).2021. WHOhttps://www.who.int/emergencies/diseases/novel-coronavirus2019. (Accessed 20/8/2021). 
Xu J, Zhang Y. 2020. Traditional Chinese medicine treatment of COVID-19. Complementary Therapy and Clinical Practice 39:101-165.

Zaki AM, Van Boheemen S,Bestebroer T, Osterhaus AD, Fouchier RAM. 2012. Isolation of a novel coronavirus from a man with pneumonia in Saudi Arabia. New England Journal of Med icine 367:1814-1820.

Zaveri M, Khandhar A, Patel S, Patel A. 2010. Chemistry and pharmacology of Piper longum L. International journal of pharmaceutical sciences review and research 5(1):67-76.

Zhang DH, Wu KL, Zhang X, Deng SQ, Peng B. 2020. In silico screening of Chinese herbal medicines with the potential to directly inhibit 2019 novel coronavirus. Journal of Integrative Medicine 18:152-158. 